\section{Dementia's demise}

\author{
Mark P. Mattson
}

Alzheimer's Research. Editor-in-chief Sarah-Jane Richards. Rapid Science. $6 / y$ r. USA \$340, elsewhere $£ 200$ (institutional); USA \$160, elsewhere $£ 90$ (personal).

AgE-RELATED neurological disorders are exacting increasingly heavy personal and financial tolls on society. Appropriately, governmental and private funding organizations are increasing their efforts to identify the causes of disorders such as Alzheimer's disease, and to develop preventive and therapeutic strategies for them. Journals devoted to a specific disease are certainly of great value in that they can focus and accelerate research efforts, an important goal of Alzheimer's Research. The editorial board consists of an excellent group of scientists whose collective expertise spans the field from molecular and cellular biology to clinical aspects of dementia. Because the key issue in the Alzheimer's field is identifying the cause(s) of nerve-cell degeneration, there is an appropriate weighting towards cellular and molecular aspects of neuronal degeneration.

Alzheimer's Research primarily publishes original research articles, with one or two short review articles per issue. A section called "Alzheimer's Focus" presents valuable brief synopses of recent high-profile articles and reports from recent meetings. Most articles published during the journal's first one-and-a-half years have addressed key issues in the field.

A large proportion of articles seem to involve independent repetitions of previously reported experiments. Although there is little glamour in confirming or contradicting earlier high-profile findings, reporting of such data provides an important avenue for consolidation of our understanding of the pathophysiological processes in Alzheimer's disease.

Articles are reviewed rapidly by contracted experts and published within 1-3 months of acceptance. There are clearly pros and cons of accelerated review and publication. On the positive side, access to recent data from other laboratories may allow researchers to accelerate their own efforts and so advance the field as a whole. On the other hand, if, through hasty review, spurious data are published, progress can actually be hindered. In the four sample issues, more than 30 per cent of the manuscripts were accepted for publication within one week of receipt, and roughly 80 per cent within one month. This clearly does not allow substantial revision of manuscripts and indicates that the articles may not contain the complete and thorough pieces of work that often arise

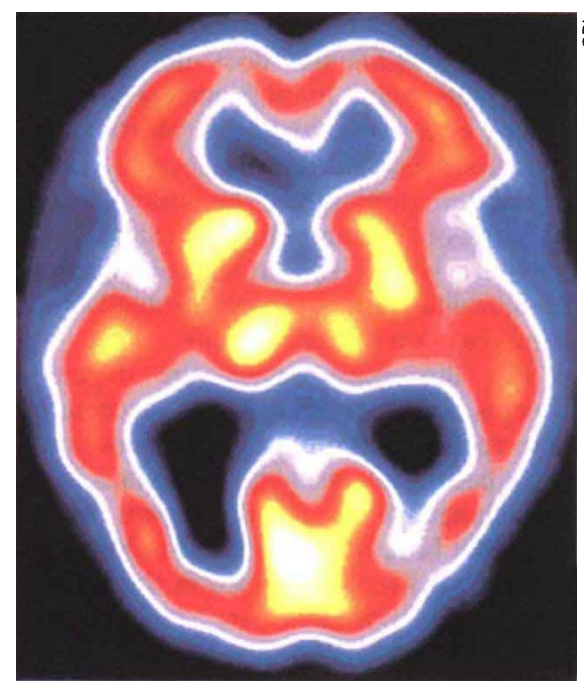

PET scan of an Alzheimer's-diseased brain.

following rigorous review.

Nevertheless, most of the articles published in the first issues seem to be of high quality and are timely, indicating that the editorial process is working and that this new journal is filling a void. The price of an institutional subscription is reasonable, but the personal rate is a bit high.

Mark P. Mattson is at the Sanders-Brown Research Center on Aging and in the Department of Anatomy and Neurobiology, University of Kentucky, Lexington, Kentucky 40536, USA.

\section{Happy reunion}

\section{Leonard I. Zon}

Nature Medicine. Editor-in-chief Barbara J. Culliton. Nature Publishing. 12/yr. $\$ 395, £ 350$ (institutional); $\$ 95, £ 90$ (personal).

Journal of Molecular Medicine. Editor-inchief D. Ganten. Springer. 12/yr. \$441, DM528 (institutional).

Journal of Biomedical Science. Editor-inchief Chuan C. Chang. Karger. 6/yr. USA $\$ 268.60$, Europe SFr340.60, elsewhere SFr355 (institutional); USA \$43.80, Europe SFr52.80, elsewhere SFr60 (personal).

MuCH of modern biology has been spawned in response to a basic question of normal human physiology or the pathophysiology of disease states. Given this origin, it is reassuring that the number of applications of molecular biology to the practice of clinical medicine increases substantially every year. Despite this apparent success story, there is a growing schism between clinicians and basic scientists because of technological and linguistic barriers. One remedy is to provide a means for the two groups to converse better. To provide such a forum for the 'reunification' of the two fields, several new journals have been created specifically to target molecular medicine.

The brightest new journal of this genre is Nature Medicine, as it generally follows the traditional style and rigour of its sister journal Nature. The layout is exciting, and flourishes with colour. "Commentaries" and "News and Views" are easily understood by clinicians and are detailed enough to please basic scientists. Book reviews appear in every issue. The editors have done a nice job maintaining the feel of a clinical journal. Most research articles appear within 3-4 months of submission. Several important articles published over the past year in Nature Medicine would traditionally have found their way into the New England Journal of Medicine. Nature Medicine will certainly be the principal journal for papers that bridge basic science and clinical medicine.

Journal of Molecular Medicine is a continuation of Klinische Wochenschrift ("The Clinical Investigator") originally published in 1864. As such, it maintains a rich tradition in blending basic research with clinical medicine. The journal particularly caters for regional submissions from Germany, but investigators from around the world do contribute. Its layout leaves room for improvement; the artwork is completely in black and white, and is occasionally underexposed. The original research articles are often short. The reviews are interesting and thorough, and are the highlight of the journal. The editors have had an excellent idea in starting a new column called "Clinical Implications", a forum for clinicians to reveal their insights into the marriage of science and medicine.

Journal of Biomedical Science similarly deals with molecular medical topics. Many submissions are from investigators in Taiwan. The artwork, reproductions and layout are done well. The journal offers rapid communication and publication; articles are often published two months after submission. Reviews are well written and timely with an emphasis on clinical-basic science issues. The original report section provides a forum for scientific correspondence; but the content of the correspondence seems only tangentially clinical, and often includes more cell biology and physiology than molecular biology.

It is no surprise that other journals such as Molecular Medicine have also appeared recently for communicating work that bridges clinical research and molecular biology. With the growing technology and advances in medicine and research, contributions to these new journals will ensure an exciting future for the union of basic science and medicine.

Leonard I. Zon is at the Howard Hughes Medical Institute, Children's Hospital, Harvard Medical School, Boston, Massachussetts 02115, USA. 